\begin{tabular}{|r|c|c|c|c|}
\hline Cuadernos de Investigación Geográfica & 2004 & N $^{\circ} 30$ & pp. 7-34 & ISSN 0211-6820 \\
\hline
\end{tabular}

(C) Universidad de La Rioja

\title{
EL MÉTODO AAR PARA LA DETERMINACIÓN DE PALEO-ELAS: ANÁLISIS METODOLÓGICO Y APLICACIÓN EN EL MACIZO DE VALDECEBOLLAS (CORDILLERA CANTÁBRICA)
}

\author{
E. SERRANO CAÑADAS (1) \\ J.J. GONZÁLEZ TRUEBA (2)
}

(1) Dpto. Geografía. Universidad de Valladolid. Po Prado de la Magdalena s/n, 47011, Valladolid. (2) Dpto. Geografía, Urbanismo y Ordenación del Territorio. Universidad de Cantabria. Avd. de los Castros s/n 39005, Santander. Fundación Marcelino Botín

Correo electrónico de contacto: serranoe@fyl.uva.es

RESUMEN: Existen diferentes métodos para la reconstrucción de paleoELAs, entre los cuales el método AAR (Acumulation Area Ratio), proporciona los resultados más fiables y es uno de los más utilizados en la actualidad. Se aplica el uso de herramientas informáticas en el cálculo de las paleo-ELAs del glaciarismo cuaternario del Macizo de Valdecebollas y se muestra la utilidad del método y algunas consideraciones sobre su aplicación y los factores que intervienen en la reconstrucción de la ELA. El cálculo de la ELA en el macizo de Valdecebollas permite aportar información que enriquece la obtenida del análisis morfológico y morfométrico. Se establece una caracterización de las condiciones ambientales de los glaciares del macizo y de las dos fases glaciares registradas: un máximo glaciar con comportamiento homogéneo de ambas vertientes (F.1) y una fase de retroceso (F.2) con comportamiento glaciar diferenciado entre el sur y el norte, orientación en la que el retroceso es entrecortado, con equilibrios de orden menor del milenio.

ABSTRACT: There are different methods for palaeo-ELAs reconstruction. The AAR method (Accumulation Area Ratio) gives the most reliable results and is one of the most frequently used methods at the moment. To calculate the "palaeoELAs" of the Quaternary glaciation of the Valdecebollas Massif the use of GIS programs is applied. It is shown the use of the method as well as some considerations about its application and the factors that play an important role in the reconstruction. The calculation of the ELA in the Valdecebollas Massif allows to provide complementary information to the morphological and morphometrical analysis. The environmental conditions of the massif in two glacial stages are established: a glacial maximum with homogeneous behaviour of their both slopes $(F .1)$ and a stage of retreat ( $F$. 2) with a different glacial behaviour between north and south faces. In this 
phase, on north face the retreat is interrupted with balances of the millennium smallest order.

Palabras clave: Línea de Equilibrio Glaciar (ELA), método A.A.R., reconstrucción paleoglaciar, macizo de Valdecebollas, Cordillera Cantábrica.

Key words: Equilibrium Line Altitude (ELA), A.A.R. method, palaeoglacier reconstruction, Valdecebollas Massif, Cantabrian Range.

Enviado en Abril de 2004

Aceptado en Junio de 2004

\section{Introducción}

El estudio de las variaciones de los glaciares en el tiempo y en el espacio en relación a los cambios ambientales se inicia hace más de dos siglos, con el precursor estudio de J. Walcher sobre los glaciares de montaña del Tirol austriaco en 1773, y se afianza con las aportaciones de los naturalistas prerrománticos (De Saussure y Ramond de Carbonnières) y decimonónicos (Humboldt, Agassiz, Desor, Vogt, Forbes, Tyndall), quienes realizan las aportaciones más destacadas. Desde finales del S. XIX se establecen en los glaciares alpinos las bases teóricas de la interrelación entre clima, condiciones ambientales y balance de masa glaciar (Simony, 1872; Höfer, 1879; Brückner, 1886,1887,1906; Richter, 1885, 1888; Kuroswki 1891; Jegerlehner, 1903; Heß, 1904; Fielding, 1908; Mougin, 1908; Marinelli, 1909; Penck y Brückner,1909).

El notable avance de las investigaciones glaciológicas en el siglo XX permitió perfeccionar las técnicas de medición y la aparición de nuevos métodos de determinación del balance de masa de los glaciares: el método glaciológico, estadístico, glacio-meteorológico, de análisis estadístico multivariable, etc. De este modo, los conocimientos adquiridos en glaciares de todo el mundo a lo largo de más de 80 años de investigación, han revelado la importancia de algunos factores climáticos y ambientales en el balance de masa de los glaciares, así como los parámetros que de forma más representativa muestran dicha interrelación.

Uno de los parámetros más útiles empleados para cuantificar el efecto del clima en los glaciares y para la caracterización ambiental de los ámbitos glaciados, es la "Altitud de la Línea de Equilibrio glaciar (ELA)". Esta se define como la altitud teórica que separa la zona de acumulación y ablación de un glaciar, donde la acumulación anual de nieve es igual a la ablación, de forma que el balance de masa es igual a 0 (Porter, 1975, 1977; Meierding, 1982; Hawkins, 1985; Kuhle, 1988; Dahl y Nesje, 1992; Nesje, 1992). La ELA, calculada con carácter anual, depende de varios factores, entre los cuales, dos se presentan como fundamentales: por un lado la acumulación anual, expresada básicamente a través de la precipitación del período invernal, y la ablación anual, que viene dada por las temperaturas del período estival. A ellos hay que sumar la importancia de la radiación como factor fundamental (Porter, 1975; Nesje, 1989; Ohmura et al., 1992; Seltzer, 1994). Junto con las características del clima regional, las condiciones geograficas de cada macizo montañoso desempeñan un papel fundamental en el balance de masa de sus glaciares. Así por ejemplo, la diferencia en el régimen térmico y pluviométrico entre un macizo de montaña de carácter continental y otro ubicado en fachada oceánica puede ocasionar notables diferencias en el balance de masa de sus glaciares, en la 
altitud a la que se sitúa la línea de equilibrio glaciar y en el tiempo de respuesta a los cambios climáticos (Ohmura et al., 1992). A su vez, otros factores como la cantidad de radiación incidente sobre la superficie glaciar, su albedo, las precipitaciones del período estival y la existencia de derrubios superficiales, influyen en el balance de acumulación y ablación en un glaciar. La topografía y las diferentes condiciones de orientación, exposición, sobrealimentación (por aludes, nieve venteada, etc), la propia geometría de la masa glaciar o la hipsometría (distribución de la superficie del glaciar en cada banda altitudinal), pueden jugar un papel fundamental en el balance de masa y en la altitud de la línea de equilibrio del glaciar. (Alhmann, 1948 ; Louis, 1955; Porter, 1975, 1977; Kuhn, 1978, 1980, 1981, 1989; Kuhn et al., 1985; Hawkins, 1985; Kuhle, 1986, 1988; Nesje, 1992; Ohmura et al., 1992; Kaser,1994; Braithwaite et al., 2002).

Tabla 1. Métodos utilizados para el cálculo de ELAs y su aplicación a la obtención de paleoELAs.

\begin{tabular}{|c|c|c|c|}
\hline MÉTODO & FUNDAMENTOS & VALORACIÓN* & REFERENCIAS \\
\hline Höfer & $\begin{array}{l}\text { La ELA se sitúa a media altura } \\
\text { entre punto más bajo del glaciar y } \\
\text { la altitud media de las paredes del } \\
\text { circo. }\end{array}$ & $\begin{array}{l}\text { Retomado y recomendado por } \\
\text { los programas internacionales de } \\
\text { la UNESCO/IASH. Poca } \\
\text { precisión, sobreestima los resultados. }\end{array}$ & $\begin{array}{c}\text { Höfer, } 1879 \\
\text { (UNESCO/IASH, } \\
1970)\end{array}$ \\
\hline $\begin{array}{l}\text { Brückner- } \\
\text { Richter } \\
\text { o AAR }\end{array}$ & $\begin{array}{l}\text { La ELA se sitúa en el límite entre } \\
\text { Área de acumulación y Área de } \\
\text { ablación. Área acumulación se } \\
\text { corresponde con: } \\
\text {-(3:1) AAR } 0.75 \text { (Brückner y Richter) } \\
\text {-(2:1) AAR } 0.67 \text { (Drygalski y } \\
\text { Machatschek) } \\
\text { - AAR } 0.6 \pm 0.05 \text { (Meir y Post) }\end{array}$ & $\begin{array}{l}\text { Actualmente muy utilizado. Se } \\
\text { obtienen los mejores resultados. } \\
\text { Factores topoclimáticos pueden } \\
\text { alterar la ELA. }\end{array}$ & $\begin{array}{c}\text { Brückner,1886, } \\
1887,1906 \\
\text { Richter, } 1885 \\
1888 \\
\text { Drygalski \& } \\
\text { Machatschek } \\
1942 \\
\text { Meir \& Post } 1962 \\
\end{array}$ \\
\hline Kurowski & $\begin{array}{l}\text { La ELA es igual a la altitud media del } \\
\text { glaciar. Considera una función linear } \\
\text { acumulación-ablación. Determinación } \\
\text { de la ELA a través de la curva hipsográ- } \\
\text { fica. Difundido como } 1: 1 \text { o AAR } 0.5 \\
\text { (Cumbre máxima+Punto más bajo } \\
\text { glaciar)/2 }\end{array}$ & $\begin{array}{l}\text { Por la dificultad del método original, } \\
\text { se utiliza la variante de la cumbre } \\
\text { más alta, más fácil, rápida y que usa } \\
\text { parámetros simples. } \\
\text { Sobreestima los resultados. }\end{array}$ & $\begin{array}{l}\text { Kuroswki, } 1891 \\
\text { Fielding, } 1908\end{array}$ \\
\hline $\begin{array}{l}\text { Heß o } \\
\text { Isohypsen- } \\
\text { methode }\end{array}$ & $\begin{array}{l}\text { Determina la ELA a través de las líneas } \\
\text { de flujo del glaciar. Pasan de convexas } \\
\text { en el frente a concavas en cabecera. } \\
\text { Considera la topografia subglaciar. }\end{array}$ & $\begin{array}{l}\text { Resultados variables y escasa preci- } \\
\text { sión. Apunta la importancia de la } \\
\text { topografía subglaciar. }\end{array}$ & $\begin{array}{l}\text { Heß, } 1904 \\
\text { Andrews y } \\
\text { Miller } 1972\end{array}$ \\
\hline $\begin{array}{l}\text { Lichtenecker } \\
\text { o "altitud má- } \\
\text { xima de las } \\
\text { morrenas } \\
\text { laterales" }\end{array}$ & $\begin{array}{l}\text { ELA determinada a partir de la altitud } \\
\text { máxima de las morrenas laterales. } \\
\text { Utiliza las bases teóricas de flujo y } \\
\text { deposición glaciar. }\end{array}$ & $\begin{array}{l}\text { Errores elevados, poca precisión y } \\
\text { dificultades. Infravalora o } \\
\text { sobrevalora la ELA. }\end{array}$ & $\begin{array}{l}\text { Lichtenecker, } \\
1938 \text { Visser, } 1938 \\
\text { Meierding, } 1982\end{array}$ \\
\hline $\begin{array}{l}\text { Glaciológico } \\
\text { Directo }\end{array}$ & $\begin{array}{l}\text { Experimentación directa. Trabajo con } \\
\text { varillas sobre el glaciar. Muy laborioso. }\end{array}$ & $\begin{array}{l}\text { Preciso, pero sólo para glaciares } \\
\text { actuales. }\end{array}$ & Ahlmann, 1948 \\
\hline $\begin{array}{l}\text { Finsterwalder } \\
\text { o Geodätische } \\
\text { meteorlg. } \\
\text { Methode }\end{array}$ & $\begin{array}{l}\text { Modifica el método Kurowski, mediante } \\
\text { una función parabólica en la relación } \\
\text { ablación-acumulación. }\end{array}$ & $\begin{array}{l}\text { Laborioso, pero con buenos } \\
\text { resultados. }\end{array}$ & Finsterwalder, 1953 \\
\hline $\begin{array}{l}\text { Altitud del } \\
\text { fondo de } \\
\text { circo glaciar }\end{array}$ & $\begin{array}{l}\text { Parámemtro fundamental: la altitud del } \\
\text { fondo del circo glaciar. Poco usado por } \\
\text { las múltiples limitaciones topográficas } \\
\text { y morfológicas. }\end{array}$ & $\begin{array}{l}\text { Errores derivados de la reconstru- } \\
\text { cción. Poco exacto, valor regional. }\end{array}$ & Pewé y Reger, 1972 \\
\hline $\begin{array}{l}\text { Umbral } \\
\text { de glaciación }\end{array}$ & $\begin{array}{l}\text { Se basa en la altitud media de las mon- } \\
\text { tañas más bajas glaciadas. }\end{array}$ & $\begin{array}{l}\text { Errores de reconstrucción, poco } \\
\text { preciso, útil para valores regionales } \\
\text { en glaciares actuales. }\end{array}$ & $\begin{array}{c}\text { Ostrem, 1966; } \\
\text { Porter, 1975, 1977; } \\
\text { Meierding, 1982 }\end{array}$ \\
\hline
\end{tabular}

\footnotetext{
* Basado en Gross et al. 1977; Meierding, 1982; Torsnes et al. 1993; Serrano, 1996; Porter, 2001.
} 
Recientemente, se ha utilizado el cálculo de paleo-ELAs para la reconstrucción de ambientes cuaternarios. Para la determinación de paleoELAs, entendiendo este concepto como la altitud a la que se encontraba la línea de equilibrio en glaciares ya desaparecidos, se estima la "Altitud de la Línea de Equilibrio Glaciar Medio" o "MELA", representativa de una fase glaciar considerada estable. Al representar unas condiciones glaciares teóricas de equilibrio, la MELA se presenta como un parámetro de gran interés, pues permite realizar comparaciones de las condiciones glaciológicas en un macizo o entre diferentes macizos de montaña a escalas locales, regionales o planetarias (Hawkins, 1985; Ohmura et al.,1992; Dahl y Nesje, 1992; Seltzer, 1994; Klein, et al. 1999; Porter, 2001). En el caso de las montañas españolas, en su mayoría deglaciadas, a excepción de varios macizos pirenaicos que albergan glaciares de reducidas dimensiones, la reconstrucción de paleoELAs puede aportar interesantes interpretaciones paleoambientales (Serrano, 1992; Serrano y Gutiérrez, 2002).

\section{Métodos de determinación de paleo-ELAs}

El interés por conocer la ELA ha conducido a establecer diferentes métodos de determinación de la altitud de la línea de equilibrio glaciar, tanto actuales como desaparecidos (Tabla 1). Por la frecuencia de utilización en los estudios de glaciarismo en áreas de montaña, hay que destacar tres métodos que hemos aplicado en el macizo de Valdecebollas.

\subsection{Método Lichtenecker o Método de la máxima altitud de las morrenas laterales}

El método de Lichtenecker (Lichtenecker, 1938) utiliza la altitud máxima de las morrenas laterales para establecer la ELA. La base teórica de este método, utilizado por Visser en el Karakorum (Visser 1938), se encuentra en las aportaciones de naturalistas como Agassiz (1840 y 1847) y Vogt (1847), y en los trabajos más específicos de Simony (1872: 258-259), Brückner (1886, 1887, 1906) y Penck y Brückner (1909). El método establece que dada la naturaleza del flujo glaciar, donde las líneas de flujo convergen hacia el centro por encima de la ELA y divergen hacia los bordes externos por debajo de la misma, las morrenas laterales, teóricamente, son depositadas sólo en la zona de ablación, es decir, justo por debajo de la ELA. De esta forma la máxima elevación de las morrenas laterales se correspondería con la ELA de la fase glaciar a la que se adscribe la morrena utilizada como base del cálculo. Este método ha dado buenos resultados para el caso de la reconstrucción de paleoELAs de glaciares pertenecientes a fases recientes, sobre todo de la Pequeña Edad del Hielo y Holoceno, y con ciertos problemas para el Tardiglaciar (Andrews, 1975; Gross et al., 1977). El problema fundamental de este método deriva de la conservación de las morrenas, de modo que si no se conservan en buen estado, la información básica para el cálculo es falsa, y la altitud de la paleoELA obtenida subestima el valor real (Hawkins, 1985; Kuhle, 1988; Nesje, 1992). Por otro lado, es un postulado teórico que la morrena se forme siempre por debajo de la ELA, y su aceptación puede implicar errores. En relación con ello, hay que tener en cuenta el error derivado de un retroceso glaciar lento y la deposición continuada de la morrena no sólo en el período de equilibrio, sino también en la fase de retroceso subsecuente, lo que sobreestimaría la altitud de la paleoELA (Kuhle, 1988; Nesje, 1992). 


\subsection{Método Kurowski}

El trabajo de L. Kurowski (Kurowski 1891; Fielding 1908) plantea que la ELA es igual a la altitud media del glaciar, y aunque en el texto original utilice el término "Schneegrenze", lo usa en el sentido moderno de ELA. El método supone un cambio lineal de ablación y acumulación con la altitud, desde el frente glaciar hasta su cabecera, que la literatura científica ha asumido como una expresión de la proporción $\mathrm{Sac} / \mathrm{Sab}$ = 1:1, o lo que es lo mismo, una relación entre el área de acumulación y el de ablación $($ AAR $)=0.5$ (Louis, 1955; Hoinkes, 1970). Para Gross et al. (1977), esta consideración no se corresponde exactamente con el método original de Kurowski, pues se excluye la "curva hipsográfica", así como la topografía subglaciar, parámetros que Kurowski utiliza, en su trabajo original, para determinar la altitud media de un glaciar. La modificación propuesta por Louis (1955) sitúa la ELA a media altura entre el punto más alto y más bajo del glaciar. Sin embargo, dada la dificultad para establecer el punto más alto alcanzado por la masa de hielo, posteriormente se ha tomado como referencia la altitud de la cumbre más alta a cuyos pies se desarrolló el glaciar reconstruido, lo que implica la elevación de la altitud media y por consiguiente de la ELA. Por la sencillez del cálculo $(E L A=($ altitud cumbre más alta + altitud del frente)/2), que tan sólo requiere dos parámetros básicos, esta variante ha sido uno de los métodos más utilizados en los estudios de glaciarismo.

Desde mediados del siglo pasado, la aplicación del método glaciológico directo en glaciares de todo el mundo, especialmente Europa y Norte América, aportó nuevos datos acerca del balance de masa y permitió determinar con más precisión sus ELAs (Ahlmann, 1948; Meir y Post, 1962; Hoinkes, 1970; Meierding, 1982). Con ello se puso de manifiesto que el método de Kurowski tiende a sobreestimar la ELA, pues la ablación disminuye con la altura más rápido de lo que aumenta la acumulación, y por tanto, la ELA, en el caso de glaciares de valle de latitudes medias, se sitúa por debajo de la altitud media del glaciar.

\subsection{Método Brückner-Richter o AAR}

El método AAR (Accumulation Area Ratio) tiene su origen en los trabajos de E. Brückner y E. Richter en los glaciares de los Alpes del este a finales del siglo XIX. Brückner (1886, 1987, 1906) asume para los glaciares de valle que el límite entre el "Firnfeld" (área de acumulación) y el "Zunge" (área de ablación) se reparte en una proporción de 3:1, o lo que es lo mismo un AAR=0.75; y que la línea resultante de dicho límite se corresponde aproximadamente con la línea de equilibrio glaciar. Brückner utiliza el término "firnlinie" en el sentido moderno de ELA. E. Richter (1885, 61), en un trabajo publicado un año antes, utilizó una proporción de 8:1, para posteriormente, siguiendo a Brückner, aplicar la proporción 3:1 (Areal Ratio 3:1), con la que el mismo Richter admitió obtener mejores resultados (Richter, 1888). El método original asumía unas condiciones teóricamente estables, debido a que, en ese momento, no se disponía de los datos cuantitativos necesarios para la elaboración de un método de tipo inductivo. El método será criticado, años más tarde, y se rechaza la proporción 3:1 considera- 
da inicialmente, al afirmar que ésta subestima el valor real de la altitud a la que se sitúa la ELA. Los nuevos estudios abogan por una proporción entre el área de acumulación y el área de ablación de 2:1 o AAR = 0,67. Esta modificiación trata de compensar algunos posibles errores del método original, derivados de la exclusión en el cálculo de la ELA de parámetros tales como son la hipsometría o la propia forma del glaciar (Drygalski y Machatschek, 1942; Klebelsberg, 1947, 1948/49).

La información aportada por la experimentación glaciológica directa, hizo posible, siguiendo el método de Brückner (1886), calcular el área de acumulación de un glaciar respecto a su área total (A.A.R., Acumulation Area Ratio) con mayor precisión, de forma que hoy es posible calcular el límite entre el área de acumulación y el área de ablación, y la altitud a la que se ubica. A partir de la experiencia obtenida en glaciares de Europa y América del norte fundamentalmente, se ha podido observar como el AAR de un glaciar varía en función de su balance de masa. De esta forma, glaciares con un AAR por debajo de 0,5 indican un balance de masa negativo, valores entre $0,5-0,8$ se corresponden con glaciares en condiciones estables, mientras que valores por encima de 0,8, muestra regímenes con balance de masa positivos (Meier y Post, 1962; Hoinkes, 1970; Andrews, 1975; Kaser, 1994). Hoy en día, se asume que para los glaciares de valle de latitudes medias, y en condiciones de equilibrio, el porcentaje del área de acumulación de un glaciar, con respecto a su área total (AAR), se sitúa en torno al 0,6 \pm 0,05 o 60\% \$ 5\% (Meir y Post, 1962; Andrews, 1975, Porter, 1975; Meierding, 1982; Hawkins, 1985).

Las ELAs derivadas de la consideración de un AAR de 0,6 0,05 han aportado los valores más aproximados, siendo éste método uno de los más utilizados en la actualidad tanto para glaciares hoy desaparecidos, como para aquellos aparatos actuales en los que se desconozca su balance de masa específico. A pesar de todo, los parámetros a considerar y las condiciones específicas de cada caso, topográficas y climáticas, pueden arrojar cifras variables (Nye, 1952; Furbish y Andrews, 1984; Hawkins, 1985; Kuhle, 1988; Dahl y Nesje, 1992; Douglas y Alastair, 1997).

\section{Glaciarismo Cuaternario y estimación de paleo-ELAs en el Macizo de Valdecebollas}

El macizo de Valdecebollas se sitúa en la vertiente meridional de la Cordillera Cantábrica (Figura 1), en la Montaña Palentina $\left(42^{\circ} 58^{`} \mathrm{~N} / 4^{\circ} 21^{`} \mathrm{~W}\right)$. Está constituido por materiales de la cobertera mesozoica, areniscas y conglomerados de características tonalidades rojizas y edad triásica. La cobertera reposa sobre las pizarras, areniscas y calizas westfalienses, que afloran en las porciones bajas de las laderas, y los circos y artesas glaciares más encajados. El macizo de Valdecebollas forma, de este modo, un cordal definido por disposiciones monoclinales con buzamientos meridionales que posibilita las culminaciones aplanadas. Presenta un amplio cordal de dirección NE-SW desde el Cueto de los Comunales (2.084) hasta el Sestil (2.063) pasando por Canaleja (2.098) y la cumbre culminante de Valdecebollas (2.143 m). Configura, así, un extenso replano en altitud conforme con las estructuras geológicas y modelado por los procesos nivales y periglaciares.

Hernández Pacheco señaló una gran parte de las morrenas presentes en el macizo de Valdecebollas y apuntó la existencia de dos fases glaciares, que atribuyó al Riss y al 


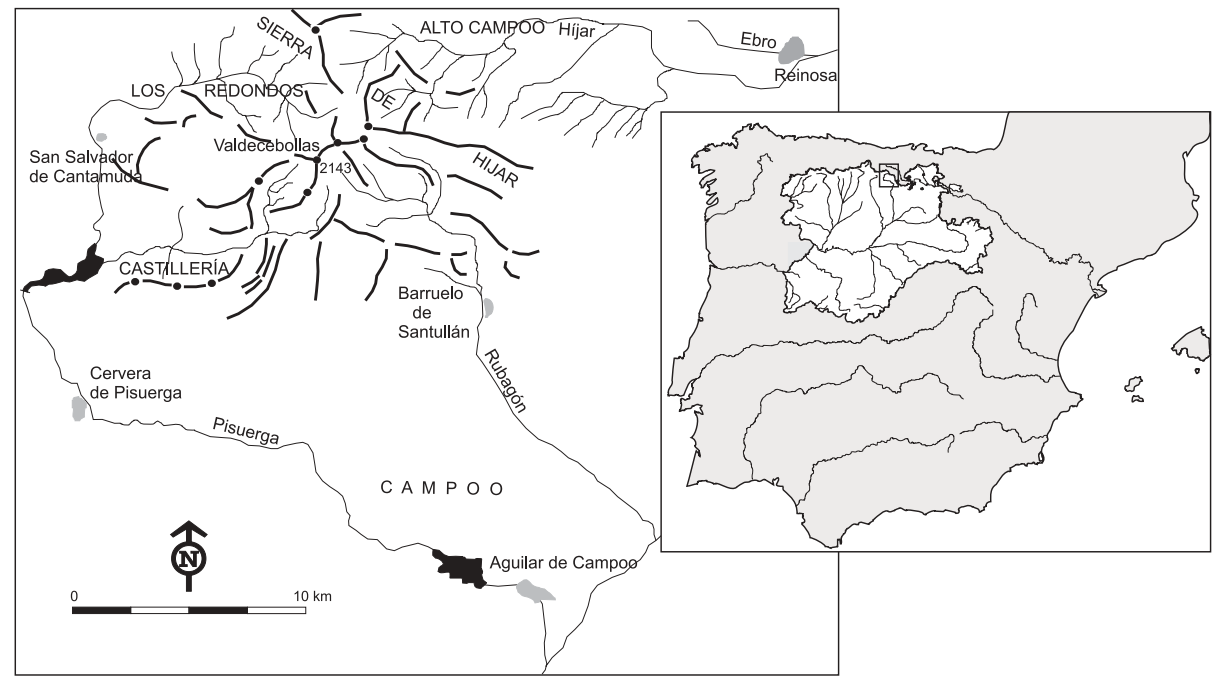

Figura 1. Localización de la zona de estudio.

Würm (Hernández Pacheco, 1944). Trabajos posteriores han señalado la imposibilidad de atribuir estos restos a dos glaciaciones diferentes (Nossin, 1959; Alonso et al., 1982; Frochoso, 1990; Frochoso y Castañón, 1998; Gutiérrez y Serrano, 1998), interpretándose como fases de la última glaciación (L.G.M., periodo isotópico 2), con múltiples pulsaciones menores (Serrano y Gutiérrez, 2000, 2002; Serrano, 2001). Además, en las cercanas montañas de Campoo se puede apreciar una última fase, aún más reciente pero independiente del retroceso de las anteriores, que permite distinguir tres familias de morrenas atribuidas a tres fases principales (Serrano y Gutiérrez, 2000, 2002).

En el macizo de Valdecebollas los cuatro glaciares pleistocenos se caracterizaron por sus pequeñas dimensiones y morfologías de circo (Figura 2 y Tabla 2).

1.- Sel de la Fuente: Es el sistema glaciar de mayor entidad, dimensiones y complejidad morfológica del macizo. Fue un glaciar con lengua de reducidas dimensiones, orientada al NW, y con una transfluencia, antiguo cauce capturado por el Pisuerga, y que trasvasaba una porción de su masa a la cuenca del Ebro. El circo es ancho, poco profundo y compuesto, con dos porciones bien diferenciadas. Al este el circo es amplio y escalonado con una sucesión de cubetas de sobreexcavación de reducidas dimensiones y los umbrales están modelados en los estratos de conglomerados. Al oeste, el circo es encajado, con escarpes verticalizados bien marcados que enlazan con la cubeta de sobreexcavación, y su morfología está determinada por la estructura. En el contacto con el paleozoico se generan amplias cubetas, separadas por un umbral glaciar sobre las calizas westfalienses. 


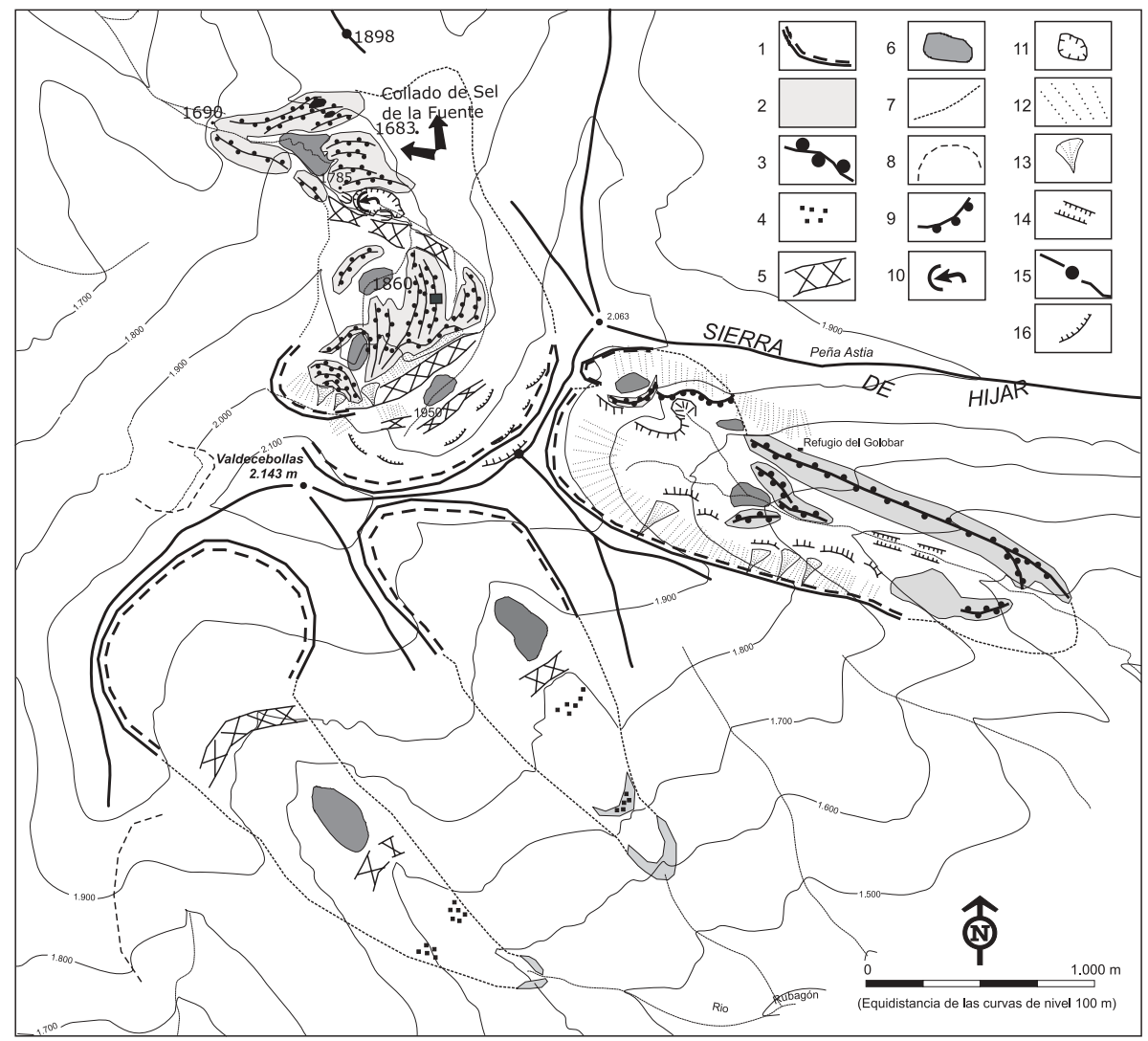

Figura 2. Esquema glaciomorfológico del macizo de Valdecebollas. 1, circo glaciar. 2, material morrénico informe. 3, morrenas. 4, bloques dispersos. 5, umbral glaciar. 6, cubeta de sobreexcavación. 7, límite del glaciar. 8, nicho de nivación. 9, morrena de nevero. 10, sumidero kárstico. 11, depresión glaciokárstica. 12, taludes de derrubios. 13, conos de derrubios. 14, incisión fluvial. 15, cordales y cumbres. 16, escarpes.

Tabla 2. Características morfométricas de los circos glaciares de Valdecebollas.

\begin{tabular}{|c|c|c|c|c|c|c|c|}
\hline $\mathrm{N}^{\mathrm{o}}$ & GLACIAR & $\begin{array}{c}\text { Anchura } \\
\mathrm{m}\end{array}$ & $\begin{array}{c}\text { Longitud } \\
\mathrm{m}\end{array}$ & $\begin{array}{c}\text { Profundidad } \\
\mathrm{m}\end{array}$ & L/A & L/P & $\mathrm{A} / \mathrm{P}$ \\
\hline 1 & Sel de la Fuente & 1375 & 875 & 283 & 0,63 & 3 & 4,8 \\
\hline 2 & Rubagón & 1250 & 1000 & 200 & 0.8 & 5 & 6,25 \\
\hline 3 & La Cárcava & 625 & 1000 & 280 & 1,6 & 3,5 & 2,2 \\
\hline 4 & Sel de Brañosera & 1000 & 250 & 280 & 1,6 & 3,5 & 2,2 \\
\hline
\end{tabular}


Este sistema glaciar depositó tres complejos morrénicos. A 1.700 m. se ubica la morrena frontal, formando un complejo compuesto por sucesivos arcos morrénicos menores y vanos intermorrénicos ocupados por lagos de obturación. A los 1.750 metros, en la cubeta inferior, se ha depositado un segundo complejo morrénico con una sucesión de siete arcos, muy próximos al anterior y que asociamos a la misma fase en sucesivas pulsaciones de retroceso. Finalmente, en la cubeta superior, entre 1.850 y 1.950 metros se suceden hasta diez arcos frontales y laterales en 900 metros de longitud, pudiendo observarse un complejo menor, bien conservado, adherido a la pared, y una sucesión de arcos laterales que alcanzan los $1.850 \mathrm{~m}$.

2.- Rubagón: Orientado al sureste, presenta un circo muy poco señalado en altura, con un encajamiento paulatino de las formas, lo que denota la escasa actividad del hielo en los amplios rellanos por encima de los 2.000 metros. El circo enlaza, mediante un umbral a favor de la presencia de estratos conglomeráticos, con un valle glaciar de pequeñas dimensiones y encajado, con una única cubeta de sobreexcavación. Destaca el afloramiento del sustrato en amplios lanchares. El circo es de tendencia longitudinal y poco profundo, sin duda por la escasa capacidad del glaciar y su adapatación a morfologías fluviales y torrenciales preglaciares. Los materiales morrénicos están representados por acumulaciones informes, deformadas por la solifluxión, y un arco morrénico hacia 1.580 metros. Las características morfológicas del complejo permiten reconstruir la máxima extensión de los hielos, pero no las distintas fases glaciares.

3. - La Cárcava: Complejo orientado al sureste, formado por un circo y artesa con materiales morrénicos. El circo se caracteriza por ser largo, estrecho y profundo, siguiendo el buzamiento de las capas y encajándose en ellas, con poco desarrollo en cabecera. Su morfología evoca, al igual que en el anterior, una adaptación a las formas fluviotorrenciales preglaciares y una escasa capacidad de remodelar las mismas. Este hecho queda confirmado por la inexistencia de circos coalescentes o horns en el macizo. A 1.580 metros se alojan materiales morrénicos laterales adosados a la ladera, y frontales, informes, que no permiten precisar la extensión del glaciar en el fondo de valle. Junto a las formas de erosión, permiten reconstruir la máxima extensión de los hielos. A 1.700 m existen acumulaciones de bloques que debido a la mala conservación de las formas impiden reconstruir con la precisión mínima la extensión de pulsaciones menores.

4.- Sel de Brañosera: Circo y artesa encajada a favor de una fractura de dirección NW-SE. El circo se orienta al norte, formando una cubeta de sobreexcavación parcialmente colmatada, pero de escasa entidad. Se trata de un circo ancho y poco profundo, con una lengua bien desarrollada, de dirección ESE.

En el circo de Brañosera sobresale la voluminosa morrena lateral, que supera el kilómetro de longitud y se alarga desde los 1.780 metros hasta el fondo del valle, donde se desdobla en dos, ya con un carácter frontal, a 1.470 metros. Señala una ocupación parcial del valle durante la fase de máxima expansión del hielo. A 1.650 metros se observan materiales morrénicos, con formas desdibujadas por la solifluxión, que señalan una fase menor, con el glaciar cobijado en el circo. 
Tabla 3. Esquema del proceso de cálculo de paleo-ELAs mediante el método AAR con ayuda de herramientas informáticas y su aplicación al glaciar del Sel de Brañosera.

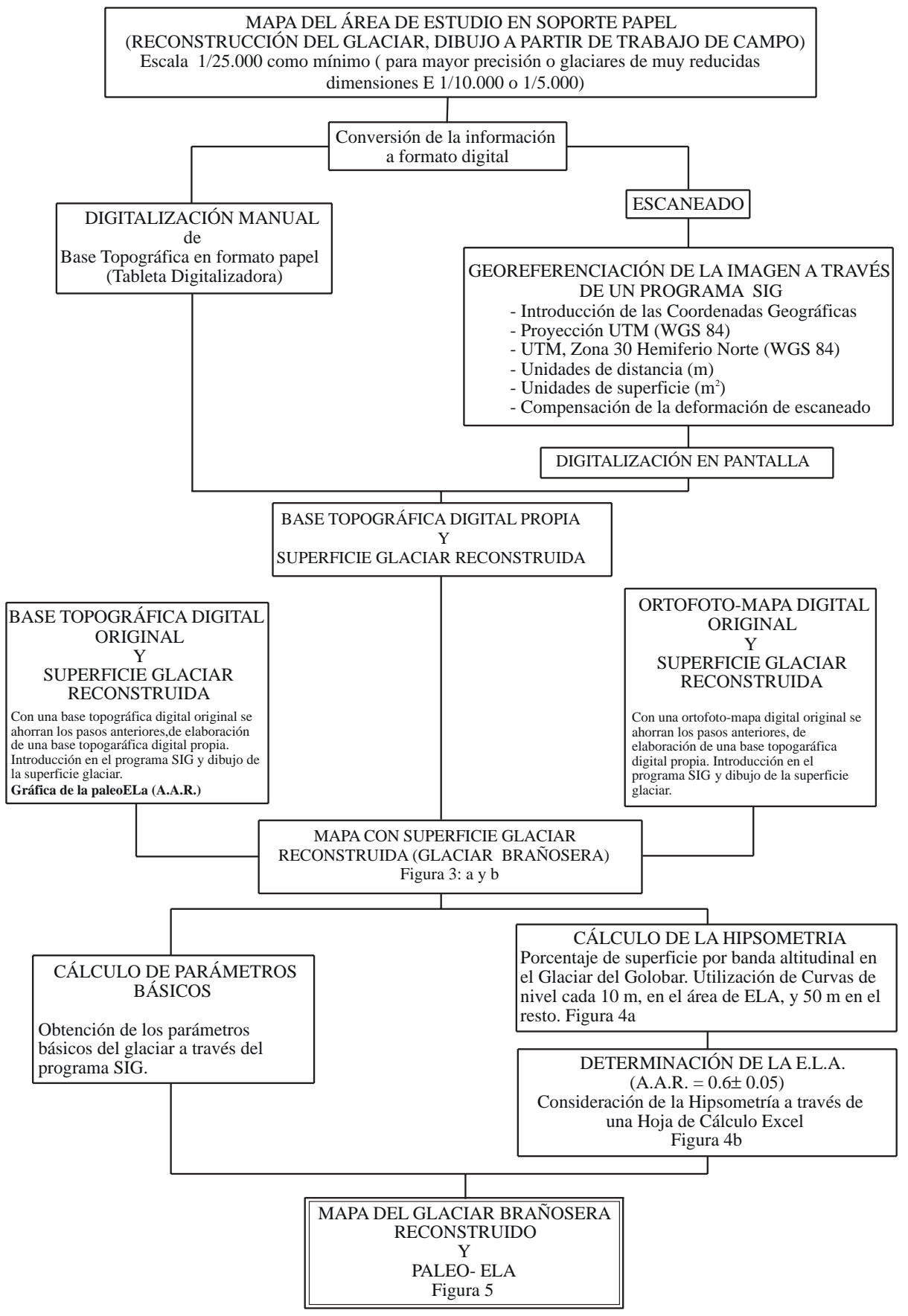




\subsection{Las paleoELAs del macizo de Valdecebollas: consideraciones metodológicas y técnicas}

La determinación de paleoELAs conlleva la existencia de fuentes potenciales de error propias del método, que deben ser tenidas en cuenta. El establecimiento preciso de la edad o fase glaciar de las formas glaciares utilizadas para la estimación de la ELA, que deben basarse en precisos estudios morfoestratigráficos; la reconstrucción paleoglaciar; los efectos orográficos; las posibles coberturas de clastos no constatadas; los gradientes de las ELAs, que conducen a confusiones o imprecisiones en el cálculo de MELAs; o los ajustes al descenso del nivel del mar para el último máximo glaciar (Porter, 2001), son algunos de ellos. Además, la determinación de las paleo-ELAs mediante el método AAR realizada a mano añadía a los errores propios del método, los derivados de la utilización de herramientas manuales. La utilización de cartografía asistida por ordenador y programas SIG en el proceso de cálculo, además de agilizarlo, reduce al mínimo los errores técnicos (Tabla 3 y Figuras 3, 4, 5).

El problema fundamental del método AAR es la reconstrucción precisa de la superficie ocupada por la masa glaciar. Dicha reconstrucción se lleva a cabo con la ayuda de las huellas morfológicas glaciares heredadas. El trabajo de campo, apoyado en la fotografía aérea, ortofotos, imágenes de satélite de alta resolución y MDTs, permite reconstruir, mediante el análisis de las formas de erosión y acumulación, la extensión y la máxima altitud ocupada por el glaciar, a través de una estimación aproximada del espesor del hielo en cabecera, lo que suele plantear las mayores dificultades. Esta fase depende de la experiencia del investigador, por lo que se han planteado aproximaciones teóricas, que tratan de ser más "objetivas", sustituyendo la delimitación del contorno a partir de la observación directa, por fórmulas matemáticas que tratan de calcular el espesor del hielo en el área de cabecera determinando el perfil longitudinal teórico del glaciar (Nye, 1952). Este tipo de cálculo ha sido retomado recientemente por Sailer et al. (1999), asistido por un SIG con base raster, pero los resultados obtenidos son válidos tan sólo para glaciares con una topografía subglaciar y una geometría glaciar simples, en la medida que el cálculo no considera estos parámetros, por otro lado fundamentales en el balance de masa de los glaciares y en la altitud a la que se localiza su línea de equilibrio glaciar.

Para la determinación de paleo-ELAs es necesario elegir una escala de análisis adecuada. Esta ha de ser, al menos, 1/25.000, y para glaciares de reducidas dimensiones (10 Has aprox. o inferior) se requieren escalas de mayor detalle, 1/10.000 o 1/5.000. En este trabajo se ha utilizado una escala de análisis 1/25.000, con una precisión de $10 \mathrm{~m}$ en el área de la ELA y $50 \mathrm{~m}$ para el resto del glaciar. Con la utilización de herramientas informáticas se agiliza el cálculo de la hipsometría y se reduce el error técnico a un $0,5 \%$ de la superficie total del glaciar, lo que supone, en condiciones constantes y homogéneas, un error de 3-5 m en la altitud de la ELA, cifra que se considera aceptable. Por contra el error derivado de la utilización de herramientas manuales ha sido estimado en torno al $2 \%$ de la superficie total, con una topografía simple, aumentando para topografías subglaciares complejas (Hawkins, 1985). 


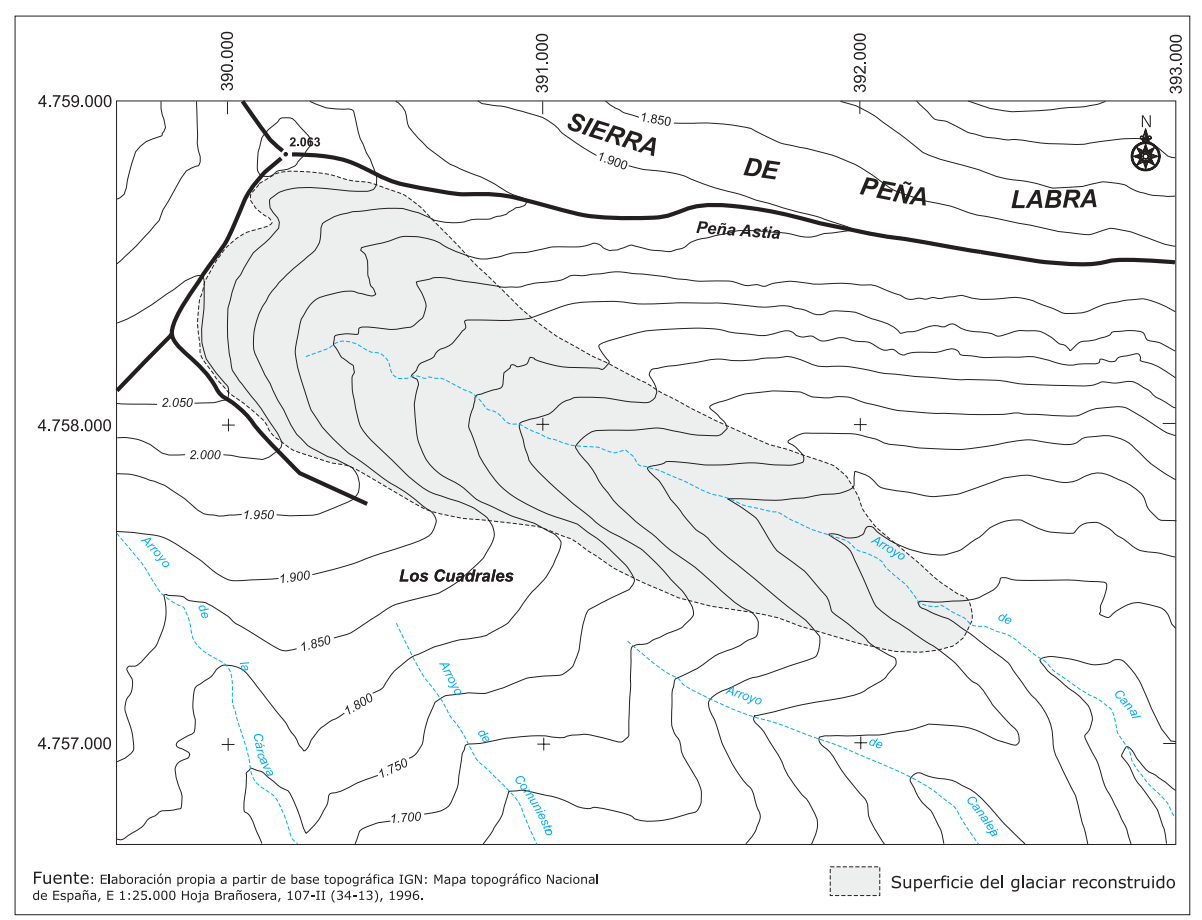

\begin{tabular}{|l|c|}
\hline Denominación del glaciar reconstruido & Glaciar del Sel de Brañosera (F.I) \\
\hline Localización Centroide UTM & X-391.142 \\
& Y-4.758.038 \\
\hline Superficie total (m2) & 1.485 .825 \\
\hline Perímetro (m) & 6.159 \\
\hline Longitud máx. (m) & 2.610 \\
\hline Anchura máx. (m) & 860 \\
\hline Altitud de la cumbre & 2.063 \\
\hline Altitud frente & 1.435 \\
\hline Desnivel cumbre-frente (m) & 628 \\
\hline Altitud máx. Masa Glaciar & 2.050 \\
\hline E.I.A. (A.A.R) & $1.705 \pm 30$ \\
\hline
\end{tabular}

Figura 3. Reconstrucción de la superficie ocupada por el glaciar del Sel de Brañosera $y$ datos básicos. 


\begin{tabular}{c|ccc}
\hline Hipsometria & Superficie $(\mathrm{m} 2)$ & $\%$ & $\%$ Acumulado \\
\hline $1.400-1.450$ & 9.135 & 0,61 & 0,61 \\
$1.450-1.500$ & 64.530 & 4,34 & 4,95 \\
$1.500-1.550$ & 105.600 & 7,1 & 12,05 \\
$1.550-1.600$ & 138.300 & 9,3 & 21,35 \\
$1.600-1.650$ & 138.900 & 9,34 & 30,69 \\
$1.650-1.700$ & 128.900 & 8,67 & 39,36 \\
$1.700-1.710$ & 26.820 & 1,8 & 41,16 \\
$1.710-1.720$ & 26.750 & 1,8 & 42,96 \\
$1.720-1.730$ & 25.220 & 1,69 & 44,65 \\
$1.730-1.740$ & 28.010 & 1,88 & 46,53 \\
$1.740-1.750$ & 29.800 & 2,02 & 48,55 \\
$1.700-1.750$ & 136.600 & 9,19 & 48,55 \\
$1.750-1.800$ & 149.700 & 10,07 & 58,62 \\
$1.800-1.850$ & 147.900 & 9,95 & 68,57 \\
$1.850-1.900$ & 162.800 & 10,95 & 79,52 \\
$1.900-1.950$ & 134.200 & 9,03 & 88,55 \\
$1.950-2.000$ & 110.300 & 7,42 & 95,97 \\
$2.000-2.050$ & 58.960 & 4,03 & 100 \\
\hline & & &
\end{tabular}

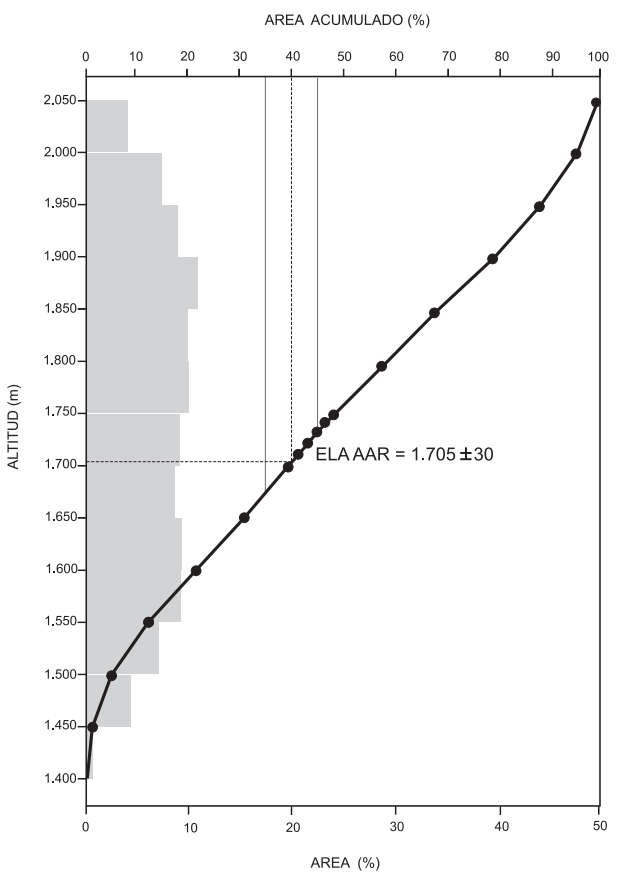

Figura 4. Gráfica de la paleoELA (A.A.R.) e hipsometría del glaciar de Sel de Brañosera. 
La topografía subglaciar, la hipsometría, la propia geometría glaciar o las condiciones orográficas (orientación, exposición, etc.) y climáticas, tanto regionales como locales (tipo de precipitación, aludes, viento, radiación, albedo, derrubios en superficie glaciar, etc.), son factores que influyen en el balance de masa de los glaciares y, por tanto, en la ELA. La utilización de un Acumulation Area Ratio de 0,6 con un margen de error de 0,05 , trata de compensar el posible error derivado de la dificultad para incluir algunos de estos factores geoclimáticos en la reconstrucción de la paleo-ELA.

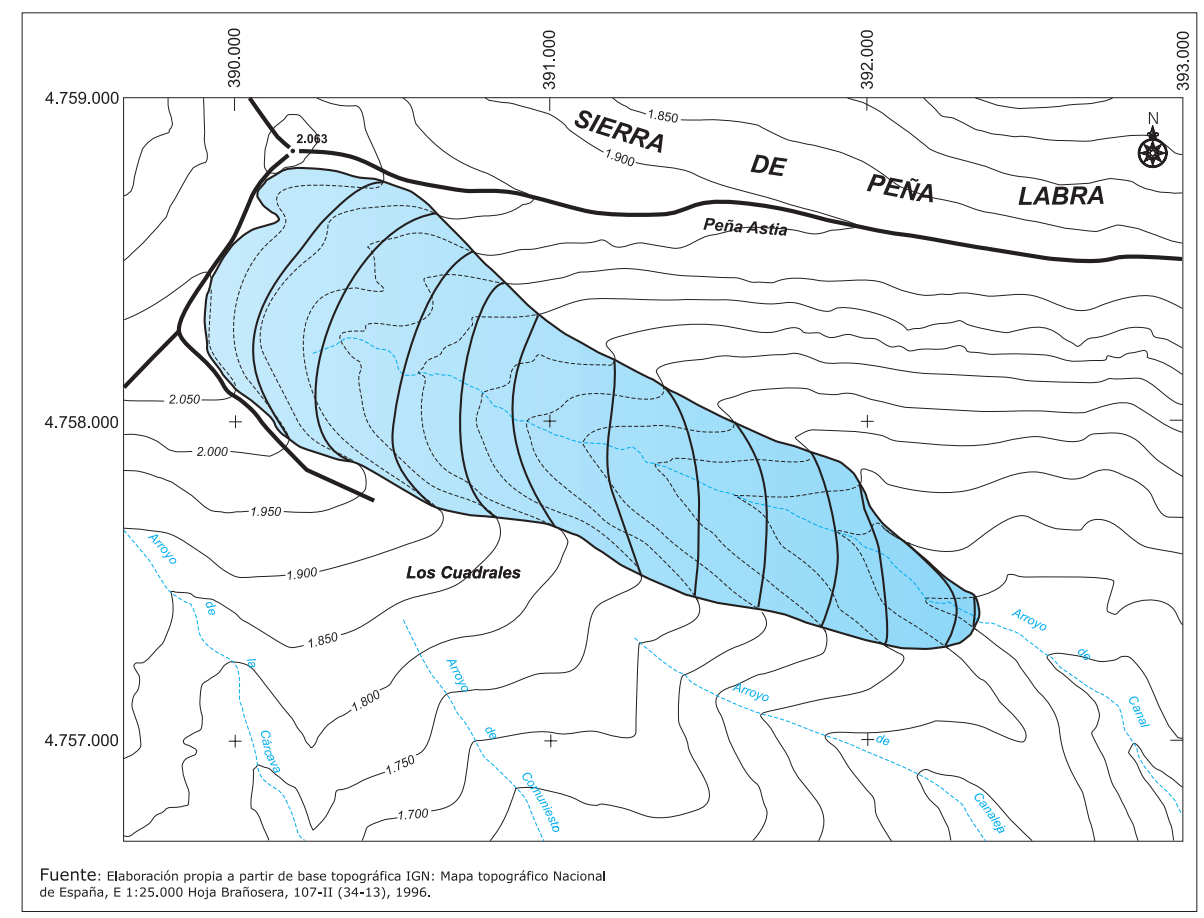

Figura 5. Reconstrucción cartográfica del glaciar de Sel de Brañosera.

Si desde el punto de vista glaciológico, la topografía subglaciar posee un papel clave en el balance de masa de los glaciares, desde un punto de vista metodológico y técnico, es un elemento fundamental a considerar en la determinación de paleo-ELAs. Los efectos de la topografía subglaciar en el proceso de cálculo de la ELA deben ser cuidadosamente evaluados. En este sentido, y en función del factor tiempo, no sólo las huellas morfológicas glaciares heredadas son susceptibles de ser borradas, sino que la propia topografía subglaciar puede ser modificada más o menos intensamente. De esta forma, la dificultad de reconstrucción del contorno glaciar, así como los errores de cálculo derivados de ello, son directamente proporcionales a la antigüedad de la fase glaciar consi- 
derada. Junto al factor tiempo, las condiciones morfoclimáticas postglaciales, o la naturaleza del sustrato, pueden jugar un papel fundamental. Las diferencias altitudinales y morfológicas entre la superficie reconstruida y la topografía subglaciar original, deben ser considerados, en la medida que el cálculo se hace en base a ésta, y no tomando como referencia el glaciar en superficie. En este sentido la utilización de una escala de análisis de alta resolución (de 10x10 m) permite detectar y corregir los errores derivados de las características topográficas subglaciares (Figura 6).

Tabla 4. Estimaciones de PaleoElas por diferentes métodos y parámetros obtenidos.

\begin{tabular}{cccccccc}
\hline GLACIAR & \multicolumn{2}{c}{ AAR ELA } & Ascenso & Pérdida & Pérdida & Kurowski & Lichtenecker \\
F.I & F.II & ELA & superficie & longitud & ELA & ELA \\
\hline Sel de la & & & & & & & \\
Fuente & $1.880 \pm 10$ & $1.887+3 /-7$ & $7 \mathrm{~m}$ & $78 \%$ & $64 \%$ & 1.929 & 1.780 \\
Rubagón & $1.862+32 /-37$ & -- & -- & -- & -- & 1.861 & -- \\
La Cárcava & $1.848+18 /-13$ & -- & -- & -- & -- & 1.861 & 1.790 \\
$\quad$ Sel de & & & & & & & 1.749 \\
Brañosera & $1.705 \pm 30$ & $1.815 \pm 15$ & $110 \mathrm{~m}$ & $57 \%$ & $43 \%$ & 1.700 \\
\hline
\end{tabular}

En el glaciar del Sel de Brañosera se puede comprobar que en función del ángulo de la ladera, por encima y por debajo de la ELA, y de los cambios de pendiente, pueden obtenerse valores altitudinales diferentes. La ELA de glaciar del Sel de Brañosera, considerando un $\mathrm{AAR}=0,6 \pm 0,05$ se sitúa a $1.705 \pm 30 \mathrm{~m}$ (en este caso el valor del margen de error es alto, dado por el ángulo de la ladera y la curva del porcentaje de área acumulado). Junto a la reconstrucción real, se han elaborado dos ejemplos hipotéticos con pendientes y cambios morfológicos variables, de forma que los resultados obtenidos muestran la influencia de la topografía subglaciar en la altitud a la que se sitúa la paleoELA. A su vez, el margen de error 0,05 o $\pm 5 \%$ del área total del glaciar reconstruido, aporta también valores "positivos" y "negativos" diferentes en función de la topografía. Si la ELA del glaciar del Sel de Brañosera, el cual posee una ladera con pendientes homogéneas, pero moderadas, se sitúa a $1.705 \pm 30 \mathrm{~m}$ (ejemplo a); en el caso de una ladera hipotética con pendiente variable (ejemplo b: rellano seguido de escarpe), la ELA se sitúa a $1.701+9 /-26$, y en el caso de una ladera hipotética con pendiente variable (ejemplo c: escarpe seguido de rellano), la ELA se sitúa a 1.740 +5/-65. Del análisis de los resultados obtenidos se deduce que los glaciares con una topografía subglaciar con pendientes homogéneas y escasa inclinación aportan los valores más fiables y menor margen de error. Por el contrario, los glaciares con una topografía subglaciar con fuerte pendiente y morfología cambiante, proporcionan valores altitudinales variables y con mayor margen de error (Figura 6).

La estimación de diferentes métodos ha permitido evaluar la respuesta de los más utilizados y analizados en este trabajo (Tabla 4). El método AAR es el más preciso porque tiene en cuenta la topografía, la hipsometría y el espesor del hielo reconstruido, de 
modo que a pesar de los errores propios del método, en ámbitos con una morfología glaciar bien marcada que posibilita la reconstrucción paleoglaciar, tiene la mayor fiabilidad. El método Kurowski establece la ELA siempre por encima del método AAR, con una sobreestimación de las paleoELAs muy variable. Del mismo modo que sucede con el método AAR, en los aparatos más simples de orientación sur, con escasas cubetas y pendientes homogéneas, los valores son más fiables y las ELAs calculadas por los métodos Kurowski y AAR, son casi coincidentes: 13 m. más alta en el caso del G. de la Cárcava (sobreestimación del 0,7\%) y 1 metro por debajo para el G. del Rubagón (subestimación del $0,05 \%$ ). Sin embargo, en los circos orientados al norte presenta altitudes muy diferentes respecto a la estimación de la ELA por el método AAR, con variaciones de $49 \mathrm{~m}$. $(2,6 \%)$ en el G. Sel de la Fuente y 44 m. (2,6\%) para el G. del Sel de Brañosera. Es por ello que puede tener un valor positivo a escala regional cuando las morfologías no permiten la reconstrucción detallada a la escala adecuada de cada aparato glaciar, pero se conocen las posiciones de los frentes y la altitud de las cumbres, y en particular para glaciares simples (de circos, sin cubetas, etc.) y de reducidas dimensiones en ámbitos glaciados marginales, propios de las montañas peninsulares. Por el contrario, el método Lichtenecker ofrece resultados muy variables. En el Sel de Brañosera sobreestima la ELA, respecto al método AAR, pero en el Sel de la Fuente y la Cárcava queda por debajo, sin que sea posible establecerla en el Rubagón. Este método, pues, es el menos fiable, de difícil aplicación en glaciares simples, con morfologías deposicionales poco definidas o con morrenas muy deterioradas por los procesos postglaciares, que impiden el reconocimiento nítido de las morrenas laterales, y ha resultado muy poco útil en el macizo de Valdecebollas.

\subsection{Caracteres y evolución del paisaje glaciar de Valdecebollas}

Las características morfológicas, los parámetros morfométricos y la información de las PaleoELAs permiten aproximarnos a las características glaciares del macizo y de las distintas fases glaciares que lo han conformado (Tabla 5). En el macizo de Valdecebollas las formas de erosión dominantes son los circos glaciares, de escaso desarrollo y adaptados a las condiciones morfoestructurales y a la morfología preglaciar. Existe una disimetría neta entre los circos orientados al norte, anchos y poco profundos, ocupados por glaciares más capaces y que remodelan las cabeceras fluviales preexistentes, y los orientados al sur, elaborados por glaciares incapaces y adaptados. Se trata, pues, de un escaso desarrollo del glaciarismo en los momentos de máxima intensidad del frío, alojado en cotas relativamente bajas -aunque significativamente más altas que otras cercanas de la Cordillera Cantábrica (p.e. Castro Valnera). Es un glaciarismo marginal, desarrollado en condiciones límite, que implica una elevada sensibilidad a los cambios climáticos. En las orientaciones meridionales los glaciares fueron incapaces de elaborar formas netas, y los circos son de morfología imprecisa.

Las formas de acumulación están bien conservadas en los complejos septentrionales, con morrenas laterales y frontales que denotan la existencia de glaciares muy pulsadores, en las que, al menos, se distinguen dos fases diferenciadas. Por el contrario, las orientadas al sur presentan formas muy desdibujadas, lo que puede indicar la menor 


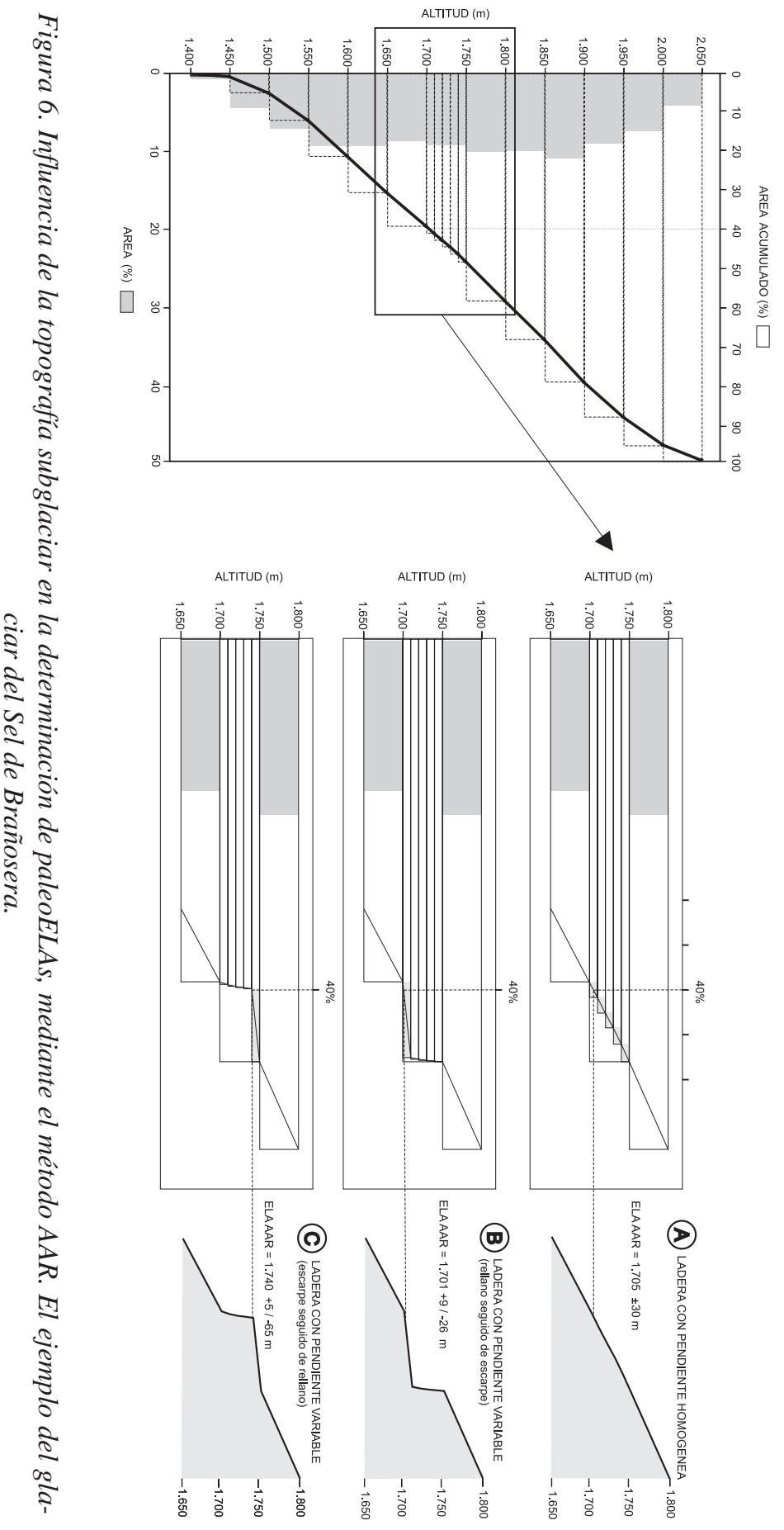




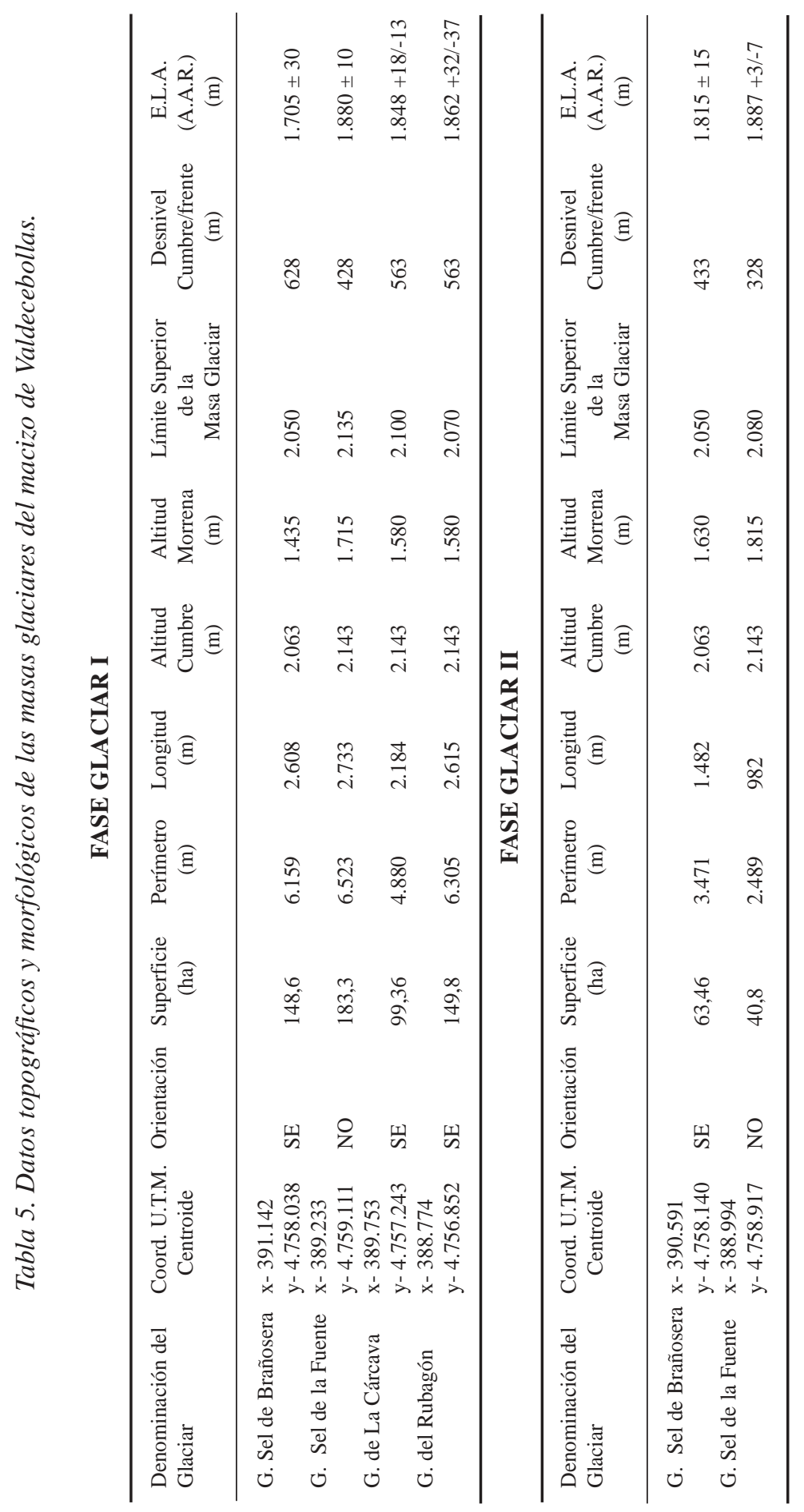


capacidad de las lenguas y su desaparición rápida y sin interrupciones. Estas formas constituyen resaltes en los circos y valles, a menudo muy deterioradas por los procesos solifluidales que impiden su utilización para la reconstrucción del glaciar.

Los glaciares de mayor superficie se sitúan al N y SE, con altitudes de la ELA muy similares y no existe un gradiente de orientación. Este hecho indica que no hay diferencias de acumulación nival entre una vertiente y otra, lo que cabe interpretar como una mayor precipitación directa al sur, o una sobrealimentación de nieve por el viento, que compense la mayor radiación de esta vertiente.

Las longitudes son similares en todos los glaciares, entre 2 y $3 \mathrm{kms}$, y si los frentes orientados al sur están muy próximos, los orientados al norte presentan una gran separación: en el Sel de Brañosera alcanza los 1.435 m, mientras en el Sel de la Fuente queda 280 metros más alto. Si tenemos en cuenta que ambos circos se alojan al norte, pero las lenguas siguen direcciones opuestas, SE en el Sel de Brañosera y NW en Sel de la Fuente, podemos deducir que es la sobrealimantación y la topografía subglaciar lo que provocaría su mayor desarrollo.

A partir de la reconstrucción de paleoELAs se pueden inferir paleotemperaturas que indiquen el descenso térmico entre distintos periodos de equilibrio y deducir algunas características ambientales. Para ello se debe aplicar un gradiente térmico y mediante la diferencia de cota estimar el descenso de temperatura en la altitud de la paleoELA más antigua. Es una estimación térmica que sólo tiene en cuenta la temperatura, despreciando los cambios de humedad y radiación. Sin embargo, son necesarios cambios importantes en las precipitaciones para que induzcan variaciones de la ELA (Seltzer, 1994; Porter, 2001), y permiten, por tanto, una estimación de los cambios térmicos en el macizo. Aplicando un gradiente de $0,6^{\circ} \mathrm{C} / 100 \mathrm{~m}$, admitido como estándar para las montañas templadas, $\mathrm{y}$, puesto que no existen glaciares en la Cordillera Cantábrica, tomando como referencia la ELA actual de los Pirineos $(3.100 \mathrm{~m})$, se pueden estimar las variaciones térmicas entre las fases 1 y 2 y entre el máximo glaciar y la actualidad. Este hecho, junto a los datos morfológicos y morfométricos permiten caracterizar las dos fases establecidas:

- Fase glaciar de Valdecebollas 1 (F.1). Corresponde la LGM, periodo de máxima extensión del hielo, que ha sido relacionado con Periodo isotópico 2 en el Pirineo meridional. Los glaciares de orientación S y SE son los más desarrollados, con mayores longitudes, y frentes más bajos, que implican glaciares de más desnivel. Los de orientación sur, Rubagón y La Cárcava, presentan los frentes a altitudes similares y un comportamiento homogéneo, adaptados a la morfología preglaciar. El glaciar del Sel de la Fuente, orientado al $\mathrm{N}$, es el más grande, pero alcanza cotas menos bajas que los orientados al sur y sureste. Este glaciar remodela los circos, pero no desarrolla una lengua como los anteriores, dividiendo su masa en una difluencia hacia la cuenca del Ebro.

La posición geográfica del macizo implica la presencia al norte de tres pantallas orográficas más elevadas, la Sierra de Peña Sagra, Sierra del Cordel y Sierra de Híjar que supondrían una reducción de las precipitaciones procedentes del norte. Por otra parte, la 

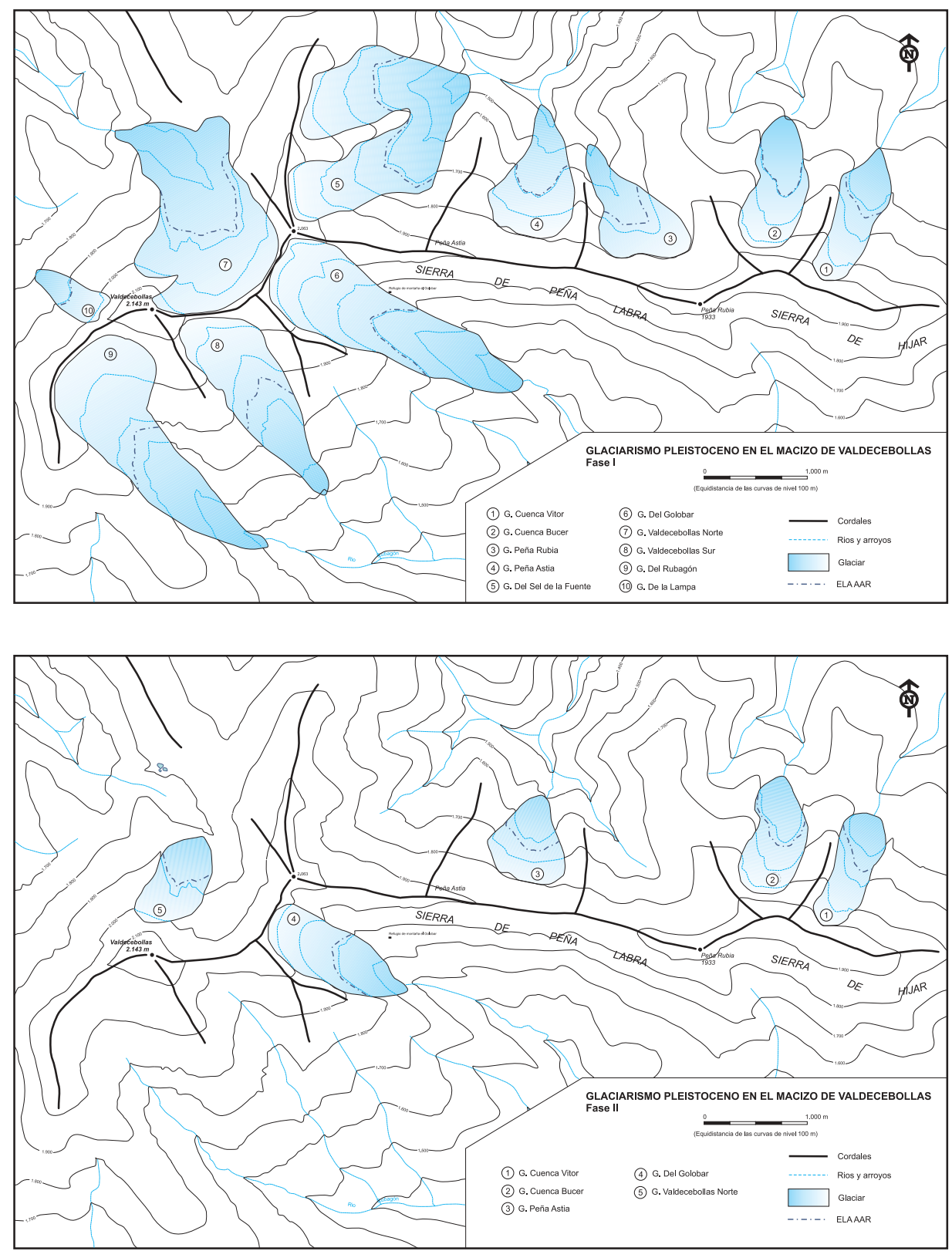

Figura 7. Evolución glaciar del macizo de Valdecebollas. 
presencia de nichos de nivación junto a la morfología de los circos indica una sobrealimentación por el viento de componente W y SW. Estos datos concuerdan con la hipótesis más tradicional (García Sainz, 1947) de la dominante de vientos del NW y sobreacumulaciones en orientaciones orientales (E y SE), o las más recientes, según las cuales el dominio de perturbaciones del oeste y suroeste por descenso de la corriente del Chorro hasta la península Ibérica aportarían la humedad y los vientos que alimentaron de nieve el macizo en ambas vertientes (Florineth y Schlüter, 2000). El glaciarismo de Valdecebollas se enmarcaría, pues, en unas condiciones definidas por precipitaciones homogéneas en ambas vertientes, de procedencia oeste y suroeste, y una sobrealimentación de los circos orientados al sur que compensaría la mayor radiación por insolación, en un ámbito continental, con la ELA AAR media para el macizo situada a $1.823 \mathrm{~m}$, mientras que mediante el método Kurowski la media queda 27 m. más alta, a $1850 \mathrm{~m}$.

- Fase glaciar de Valdecebollas 2 (F.2). Corresponde a la fase de retroceso y desaparición de los glaciares en el macizo, durante el mismo periodo que F1 (P.I.2). Es un retroceso paulatino en los circos orientados al sur y con pulsaciones menores en los orientados al norte. Sobre todo en el Sel de la Fuente será muy pulsador durante todo el retroceso, abandonando hasta 19 arcos morrénicos menores. En este período existe un gradiente de orientación, derivado del hecho de que no existen morrenas bien conservadas al sur, mientras al norte se han generado arcos múltiples asociados a un retroceso entrecortado y con periodos de equilibrio más señalados. Dicho comportamiento lo interpretamos como la existencia de más alimentación en los circos septentrionales o ausencia de sobrealimentación en los meridionales. Este hecho, unido a una menor insolación de la umbría y las consiguientes temperaturas más bajas, posibilitó la persistencia del hielo y una mayor sensibilidad de los glaciares, de reducidas dimensiones, a los cambios térmicos o de precipitaciones. Al sur, la fusión sería continua, pues los cambios en la alimentación nival serían compensados por el incremento de la radiación y la elevación de las temperaturas. La ELA se situaría a $1851 \mathrm{~m}$. al norte, mientras los glaciares de la vertiente meridional desaparecerían como consecuencia de las desfavorables condiciones ambientales. El ascenso de la ELA representaría 28 m. al N, y más de 270 m. al S, denotando ya un comportamiento dinámico muy disimétrico.

El comportamiento diferenciado de los circos entre las vertientes indica un cambio en las condiciones ambientales del macizo respecto a la fase anterior. El ascenso de la ELA es amplio en el Sel de Brañosera, donde queda cobijado al norte de modo definitivo, perdiendo la mitad de su superficie y casi de longitud (Tabla 4). Es menor en Sel de la Fuente, casi imperceptible, aunque sin embargo pierde un $77 \%$ de su superficie, más de la mitad de su longitud (64\%) y queda también cobijado totalmente al norte. La retirada entrecortada, que continúa hasta momentos muy recientes, evoca un comportamiento diferente del glaciar, con una alta sensibilidad al incremento de la alimentación nival, ya sea por el aumento de precipitaciones o el descenso de las temperaturas. Ahora los glaciares del norte están mejor alimentados que los del sur y estos últimos, con el ascenso de temperaturas, se funden. Entre la fase I y II el ascenso de temperaturas calculado a partir del ascenso de la ELA es mínimo, $0,6^{\circ} \mathrm{C}$ en el Sel de Brañosera y $0,1^{\circ} \mathrm{C}$ en el Sel de La Fuente. Este hecho indica que no son fases glaciares con características 
térmicas muy diferenciadas e interpretamos la $\mathrm{F} 2$ como un periodo de retroceso del máximo glaciar (F1), que refleja los cambios ambientales que condujeron a la deglaciación del macizo, pero no un periodo glaciar largo y con caracteres propios (Figura 7).

Entre el máximo glaciar y la actualidad se establece un ascenso de las temperaturas menor de $7^{\circ} \mathrm{C}$, pero próximo a él. Este ascenso térmico debió ser muy fragmentado y acompañado de cambios de humedad, condiciones necesarias para producir los constantes equilibrios y retrocesos que depositaron los sucesivos arcos morrénicos. Un glaciar de tan reducidas dimensiones es muy sensible a los cambios ambientales y es posible que con un ascenso térmico más o menos continuo, las variaciones de precipitaciones implicaron respuestas rápidas, con retrocesos y periodos de equilibrio, en glaciares muy dinámicos que respondieron construyendo arcos morrénicos sucesivos en una escala temporal próxima o menor del milenio.

\section{Conclusiones}

El método AAR es el más apropiado para la determinación de paleo-ELAs, en comparación con otros métodos analizados. Tras él, el método kurowski muestra una aplicabilidad útil en sistemas glaciares sencillos y con dimensiones reducidas, tendiendo a sobreestimar la ELA. En la aplicación del método AAR hay que considerar múltiples factores y la exclusión en el proceso de cálculo de algunos de ellos, obliga a asumir las limitaciones propias del método. La utilización de herramientas informáticas y una escala de análisis adecuada, minimizan los errores técnicos, dejando el margen de error tan sólo para los derivados del método. Los errores técnicos estimados, en torno a los 3-5 metros para la ELA mediante el cálculo AAR, son despreciables para la reconstrucción de condiciones ambientales frías del pasado, si bien es necesario considerar la topografía subglaciar de cada aparato. La consideración de las causas de ese margen de error puede ayudar al investigador, sin poseer datos cuantitativos, a orientar el resultado final del cálculo hacia valores altitudinales más reales. Este método se muestra, pues, apropiado para obtener más información de las condiciones y los cambios ambientales sucedidos durante las fases glaciares y la deglaciación en medios de montaña, tanto a escala de macizo como de cordillera o regional.

La información obtenida del cálculo de las ELAs en el macizo de Valdecebollas, para las diferentes fases glaciares, enriquece la aportada por el análisis morfológico y morfométrico. En el macizo de Valdecebollas se aprecia un máximo glaciar con un comportamiento homogéneo entre las vertientes septentrional y meridional, indicando la ausencia de diferencias de acumulación nival entre una vertiente y otra. Este hecho lo interpretamos como una mayor precipitación directa al sur, o una sobrealimentación de nieve por el viento, que compensa la mayor radiación de esta vertiente. Estudios en los macizos y sierras del entorno permitirán concretar esta apreciación a escala regional. Una segunda fase se caracteriza por el desigual comportamiento entre vertientes y una fuerte disimetría; al norte, glaciares de reducidas dimensiones y en condiciones límite, muy pulsadores dada su sensibilidad a los cambios climáticos y ambientales, y al sur, desaparición completa de las masas glaciares. Este comportamiento indica la desaparición de las con- 
diciones del máximo glaciar durante el proceso de deglaciación. La sensibilidad y multiplicidad de arcos durante el retroceso en el Sel de la Fuente, ha impedido constatar la existencia de una fase última, registrada en macizos cercanos, y la reconstrucción de la evolución ambiental de la misma.

La escasa diferencia térmica entre la fase I y II, $0,6^{\circ} \mathrm{C}$ en el caso de Sel de Brañosera, y $0,1^{\circ} \mathrm{C}$ en Sel de la Fuente, proporcionada por el ascenso de las paleoELAs, permiten adscribir las dos fases analizadas al L.G.M., que ha sido correlacionado con el P.I.2 del Pirineo meridional. La F. I se correspondería con el momento de máxima extensión de los hielos, con la MELA situada a 1.823 m., mientras que F. II sería una fase de retroceso, dentro del mismo período, con la MELA a $1851 \mathrm{~m}$., para las orientaciones norte. El ascenso de la MELA ha sido estimado en $28 \mathrm{~m}$. al N, y más de $270 \mathrm{~m}$. al S, mostrando ya un comportamiento dinámico muy disimétrico, entre ambas orientaciones. El análisis de la evolución glaciar del Macizo de Valdecebollas, ha mostrado la sensibilidad de los glaciares de reducidas dimensiones a los cambios climáticos y ambientales, así como la utilidad de la determinación de las paleoELAs para la reconstrucción paleoambiental de ámbitos glaciados marginales.

\section{Agradecimientos}

Deseamos agradecer a la Fundación Marcelino Botín la ayuda para la estancia en el Instituto de Geografía y Estudios Regionales de la Universidad de Viena, donde se ha recogido y tratado buena parte de la documentación utilizada.

\section{Referencias bibliográficas}

Agassiz, L. (1840). Untersuchungen über die Gletscher. Ed. Nicolet, Neuchatel.

Agassiz, L. (1847). Nouvelles études et experiencies sur les glaciers actuels: leur structure, leur progresión et leur activité physique sur le sol. Victor Masson Ed., 598 pág., París.

Ahlmann, H.W. (1948). Glaciological research on the North Atlantic Coasts. Royal Geographical Society. (Research series 1), London.

Alonso, F.; Arenillas, M. y SAEnZ, C. (1982). La morfología glaciar en las Montañas de Castilla La Vieja y León. En: I Congreso de Geografía de Castilla-León, Concejo General de Castilla y León, pp. 23-41, Burgos.

ANDREWS, J.T. (1975). Glacial systems. An aproach to glaciers and their environments. Duxbury Press, pp.191. North Scituate.

Braithwaite, R.J.; Zhang, Y. y RAPER, S.C.B. (2002). Temperature sensitivity of the mass balance of mountain glaciers and ice caps a climatological characteristic. Zeitschrift für Gletscherkunde und Glazialgeologie, 38-1: 35-61, Innsbruck.

BRÜCKNER, E. (1886). Die Hohern Tauern und ihre Eisbedeckung. Zeitschrift des Deutsch-Österreichische Alpenvereins, 17: 163-187, Innsbruck.

BRÜCKNER, E. (1887). Die Höhe der Schneelinie und ihre Bestimmung. Meteorologische Zeitschrift, 4: 31-32. 
BRÜCKNER, E. (1906). Die Höhe der Firnlinie am Hüfigletscher und die Methode der Bestimmung der Höhe der Firnlinie im allgemeinen. Vierteljahrsschrift d. Naturf. Ges. Zürich, 51: 50-54, Zürich.

DAHL, S.O. y NeSJE, A. (1992). Paleoclimatic based on equilibrium line altitude depressions of reconstructed Younger Dryas and Holocene cirque in Inner Nordfjord, western Norway. Paleogeography, Palaeoclimatology and Palaeoecology, 94: 87-97.

Douglas, B. y Alastair, G. (1997). Calculating equilibrium-line altitudes of former glaciers by the balance ratio method: a new computer spreadsheet. Glacial Geology and Geomorphology, tn 01.7 pág.

Drygalski, E. y MachatscheK, F. (1942). Gletscherkunde. Enzyklopädie der Erdkunde, 261 pág., Wien.

FIELDING, H. (1908). A proof of Kurowski rule for determining the height of the névéline on glaciers. Zeitschrift für Gletscherkunde und Glazialgeologie, Kleine Mitteilungen: 142-145.

FinsterwaldER, R. (1953). Die zahlenmaßige Erfassung des Gletscherrückganges an Ostalpengletschern. Zeitschrift für Gletscherkunde und Glazialgeologie 2, 2: 189 239, Innsbruck.

Florineth, D. y SchlÜTER, C. (2000). Alpine evidence for atmospheric ciculation patterns in Europe during the Last Glacial maximum. Quaternary Research, 54, 295 308.

Frochoso, M. (1990). Geomorfología del Valle del Nansa. Universidad de Cantabria, Santander.

Frochoso, M. y Castañón, J.C. (1998). El relieve glaciar de la Cordillera Cantábrica. En: Las huellas glaciares de las montañas españolas (Gómez Ortiz, A. y Pérez Alberti, A., Eds.). Ediciones Universidad de Santiago de Compostela, pp. 65-138, Santiago de Compostela.

FurBish, D.J. y ANDREws J.T. (1984). The use of Hypsometry to indicate long-term stability and response of valley glaciers to changes in mass transfer. Journal of Glaciology, 30: 199-211.

GARCíA SAINZ, L. (1947). El clima de la España Cuaternaria y los factores de su formación. Universidad de Valencia. Valencia.

Gross, G.; Kerschner, H. y PAtzelt, G. (1977). Methodische Untersuchungen über die Schneegrenze in alpinen Gletschergebieten. Zeitschrift für Gletscherkunde und Glazialgeologie, 12, 2: 223-251, Innsbruck.

Gutiérrez, A. y Serrano, E. (1998). El yacimiento del Paleolítico Medio de la "ermita del Abra" (Campoo de Suso, Cantabria). Aproximación cultural, cronológica y geomorfológica. Cuaternario y Geomorfología, 12: 27-39.

HAWKINS, F.J. (1985). Equilibrium line altitude and paleoenvironment in the Merchants Bay area, Baffin Island, N.W.T. Canada. Journal of Glaciology, 31-109: 205-213. 
Hernández Pacheco, F. (1944). Fisiografía, geología y glaciarismo cuaternario en las montañas de Reinosa. Memorias de la Real Academia de Ciencias Físicas Exactas y Naturales, serie Ciencias Naturales. Madrid.

Heß, H. (1904). Die Gletscher. Fr. Wieweg und Sohn. 426 pág, Braunschweig.

HÖFER, H. (1879). Gletscher und Eiszeitstudien. Sitzungsber.D.Akad.d.Wiss.Wien, Math.-Nat. Kl. 1, 79: 331-367, Wien.

HoinKes, H. (1970). Methoden und Möglichkeiten von Massenhaushaltsstudien auf Gletschern. Zeitschrift für Gletscherkunde und Glazialgeologie, 6-2: 37-90, Innsbruck.

Jegerlehner, J. (1903). Die Schneegrenze in den Gletschergebieten der Schweiz. Gerlands Beiträge zur Geophysik, 5: 486-567.

KAser, G. y Kerschner, H. (1994). Equilibrium line altitudes of Younger Dryas glaciers in the Alps as paleoclimatic information sources. Annales Geophysicae, 12-2: 386.

Klebelsberg, R. (1947). Die heutige Schneegrenze in den Ostalpen. Mitt. d. Naturwiss. Medizinischen Vereins, 47: 9-32, Innsbruck.

KleBelsberG, R. (1948/49). Handbuch der Gletscherkunde und Glazialgeologie. Bd. I. (1948), Bd. II. (1949), Wien Springer, 1028 pág., Wien.

Klein, A.G.; Seltzer, G.O. y Isacks, B.L. (1999). Modern and last local glacial maximum snowlines in the central Andes of Perú, Bolivia and northern Chile. Quaternary Science Review, 18: 63-84.

Kunle, M. (1986). The Upper Limit of Glaciation. GeoJournal, 13, 4: 331-346, Dordrecht.

KuHLE, M. (1988). Topography as a fundamental element of glacial systems. A new approach to ELA calculation and typological classification of paleo-and recent glaciations. GeoJournal, 17,4: 545-568, Dordrecht.

KuHn, M. (1980). Die Reaktion der Schneegrenze auf Klimaswankungen. Zeitschrift für Gletscherkunde und Glacialgeologie, 16, 2: 241-254, Innsbruck.

Kunn, M. (1981). Climate and Glaciers. En: Sea Level, Ice and Climatic Change. 131 Symposium International Association of Hydrological Sciences Publication (1979), pp 3-20, Camberra.

Kunn, M. (1989). The response of the equilibrium line altitude to climate fluctuations: Theory and observations. En Glacier Fluctuations and Climate Change (Oerlemans, J. ed.). Kluwer, pp. 407 - 417, Dordrecht.

KuRowsKi, L. (1891). Die Höhe der Schneegrenze mit besonderer Berücksichtigung der Finsteraarhorngruppe. Geographische Abhandlungen, 5, 1: 115-160.

LichtenECKER, N. (1938). Die gegenwärtige und die eiszeitliche Schneegrenze in den Ostalpen. En: Verhandl.d.III. Intern.Quartär-Konferenz (1936), pp141-147. Wien.

LouIs, H. (1955). Schneegrenze und Schneegrenzbestimmung. Geographisches Taschenbuch, 1954/55: 414-418. 
Marinelli, O. (1909). Il limite climatico delle nevi nel gruppo del M. Canin (Alpi Giulie). Zeitschrif für Gletscherkunde und Glazialgeologie: 334-346, Innsbruck.

MEIR, M.F. y Post, A.S. (1962). Recent variations in mass net budgets of glaciers in western North America. En: Comm. des Neiges et des Glaces. Union Geodesique et Geophysique Internationale. Assoc. Internat. d'Hydr. Scient, pp 63-77, Oberqurgl.

MEIERDING, T.C. (1982). Late Pleistocene glacial equilibrium line altitudes in the Colorado Front Range: a comparison of methods. Quaternary Research, 18, 3 : 289310 .

Mougin, P. (1908). L'altitude de la ligne des neiges et son relèvement actuel dans les Alpes de la Savoie. Zeitschrif für Gletscherkunde und Glazialgeologie, 4: 285-292, Innsbruck.

NesJe, A. (1992). Topographical effects on the Equilibrium-Line Altitude on Glaciers. GeoJournal, 27, 4: 383-391, Dordrecht.

Nossin, J.J. (1959). Geomorphological aspects of the Pisuerga drainage area in the Cantabrian Mountains (Spain). Leidse Geologishe Mededelingen, 24 :283-406.

NyE, J.F. (1952). A comparison between the theoretical and the measured long profile of the Unter-Aar glacier. Journal of Glaciology, 2: 103-107.

Ohmura, A., Kasser, P. y FunK, M. (1992). Climate at the equilibrium line of glaciers. Journal of Glaciology, 38, 130: 397-411.

Østrem, G. (1966). The height of the glaciation limit in southern British Colombia and Alberta. Geografiska Annaler, 48A: 126-138.

Penck, A. y BrüCKner, E. (1909). Die Alpen im Eiszeitalter. 3 B., 1199 pág., Leipzig.

PÉwÉ, T.L. y REGER, R.D. (1972). Modern and wisconsinan snowlines in Alaska. En: Procceding of the XXIV International Geological Congress, secc12, pp. 187-197.

PORTER, S.C. (1975). Equilibrium line altitudes of Late Quaternary glaciers in the Southern Alps, New Zealand. Quaternary Research, 5: 27-47.

PORTER, S.C. (1977). Present and past glaciation threshold in the Cascade Range, Washington USA: Topographic and climate control, and paleoclimatic implications. Journal of Glaciology 18, 78: 101-116.

PORTER, S.C. (2001). Snowline depression in the tropics during the last glaciation. Quaternary Science Review, 20: 1067-1091.

RICHTER, E. (1885). Beobachtungen an den Gletschern der Ostalpen. II. Die Gletscher der Ötzhaler Gruppe im Jahre 1883. Zeitschrift des Deutschen und Österreichischen Alpenvereins 16: 54-65, Innsbruck.

Richter, E. (1888). Die Gletscher der Ostalpen. J. Engelhon, Stuttgart.

SAILER, R.; Kerschner, H. y Heller, A. (1999). Three-dimensional reconstruction of Younger Dryas glaciers with a raster-based GIS. Glacial Geology and Geomorphology. 24 pág.

Seltzer, G.O. (1994). Climatic interpretation of alpine snowline variations on millennial time scales. Quaternary Research, 41: 154-159. 
SERrANO, E. (1996). Líneas de equilibrio glaciar, glaciares rocosos y paleoambiente postglaciar en la alta montaña pirenaica (macizo de Panticosa, Pirineo aragonés). En: Dinámica y Evolución de Medios Cuaternarios (Pérez Alberti A., Martini P., Chesworth W. \& Martinez Cortizas A. Ed.). Ediciones Universidad de Santiago de Compostela, pp. 157-170, Santiago de Compostela.

Serrano, E. y Gutiérrez, A. (2000). Las huellas de la última glaciación en Campoo. Cuadernos de Campoo, 20: 4 -14, Reinosa.

SERrAno, E. y GutiérRez, A. (2002). El glaciarismo pleistoceno en la vertiente meridional de la Cordillera Cantábrica (montañas de Palencia, Cantabria y Burgos). En: Geomorfología y Paisaje. Guía de excursiones. VII Reunión Nacional de Geomorfología. S.E.G-Depto. Geografía Universidad de Valladolid, pp. 91-161, Valladolid.

Simony, F. (1872). Gletscher und Flußschutt als Object wissenschaftlicher Detailforschung. Mitt. d. k.k. Geogr. Ges. in Wien 15: 252-275, 327-333, Wien.

Torsnes, I.; Rye, N. y Nesje, A. (1993). Modern and Little Ice Age equilibrium line altitudes on outlet valley glaciers from Jostedalsbreen, western Norway: an evaluation of different approaches to their calculation. Artic and Alpine Research, 25: 106-116.

VISSER, P.C. (1938). Wissenschaftliche Ergebnisse der Niederländischen Expeditionen in den Karakorum und die angrenzenden Gebiete in den Jahren 1922-1935. II. Glaziologie. 216 pág., Leiden.

Vogt, C. (1847). Agassiz geologische Alpenreisen. Hrsg v. C. Vogt, 2, Aufl. Frankfurt/M. 672 pág., Frankfurt. 
